\title{
Night-time care routine interaction and sleep disruption in adult cardiac surgery
}

\author{
Jesus M Casida PhD, RN, APN-C, Assistant Professor ${ }^{1}$ (D) Jean E Davis PhD, RN, FAAN, \\ Paul J. McKee Senior Associate Dean for Research and PhD Program Director ${ }^{2}$ । \\ Aaron Zalewski BSN, RN, Staff RN ${ }^{3}$ | James J Yang PhD, Associate Research Scientist ${ }^{1}$
}

${ }^{1}$ University of Michigan School of Nursing, Ann Arbor, MI, USA

${ }^{2}$ Barnes-Jewish College Goldfarb School of Nursing, St. Louis, MO, USA

${ }^{3}$ Progressive Care Unit, University of Wisconsin Hospitals and Clinics, Madison, WI, USA

\section{Correspondence}

Jesus M Casida, University of Michigan School of Nursing, Ann Arbor, MI, USA. Email: jcasida@umich.edu
Aims and Objectives: To explore the context and the influence of night-time care routine interactions (NCRIs) on night-time sleep effectiveness (NSE) and daytime sleepiness (DSS) of patients in the cardiac surgery critical-care and progressive-care units of a hospital.

Background: There exists a paucity of empirical data regarding the influence of NCRIs on sleep and associated outcomes in hospitalised adult cardiac surgery patients.

Methods: An exploratory repeated-measures research design was employed on the data provided by 38 elective cardiac surgery patients (mean age $60.0 \pm 15.9$ years). NCRI forms were completed by the bedside nurses and patients completed a 9-item Visual Analogue Sleep Scale (100-mm horizontal lines measuring NSE and DSS variables). All data were collected during postoperative nights/days (PON/POD) 1 through 5 and analysed with IBM SPSS software.

Results: Patient assessment, medication administration and laboratory/diagnostic procedures were the top three NCRIs reported between midnight and 6:00 a.m. During PON/POD 1 through 5, the respective mean NSE and DSS scores ranged from $52.9 \pm 17.2$ to $57.8 \pm 13.5$ and from $27.0 \pm 22.6$ to $45.6 \pm 16.5$. Repeatedmeasures ANOVA showed significant changes in DSS scores $(p<.05)$. NSE and DSS were negatively correlated $(r=-.44, p<.05)$, but changes in NSE scores were not significant $(p>.05)$. Finally, of 8 NCRIs, only 1 (postoperative exercises) was significantly related to sleep variables $(r>.40, p<.05)$.

Conclusion and relevance to clinical practice: Frequent NCRIs are a common occurrence in cardiac surgery units of a hospital. Further research is needed to make a definitive conclusion about the impact of NCRIs on sleep/sleep disruptions and daytime sleepiness in adult cardiac surgery. Worldwide, acute and critical-care nurses are well positioned to lead initiatives aimed at improving sleep and clinical outcomes in cardiac surgery.

KEYWORDS

acute care, adult nursing, cardiovascular, care activities, critical care, sleep disturbance, sleep quality, surgical nursing 


\section{1 | BACKGROUND}

Sufficient sleep is a basic human need essential for restoring the body's homeostatic function altered by illness, injury or surgery (Buysee, 2014; Friese, 2008). However, insufficient sleep is common in patients who have undergone cardiac surgery, despite the remarkable advancements in surgical techniques (Bojar, 2011) and the availability of private rooms in the cardiac surgery critical-care and progressive-care units of hospitals (Thompson et al., 2012). In fact, sleep disruption remains a major problem reported by patients and is highly prevalent in adult cardiac surgery. Studies show that over $80 \%$ of adults, 30 years and older, had sleep disruptions during the first week after cardiac surgery. In these studies, nearly $70 \%$ of adults who had coronary artery bypass and/or heart valve surgery continued to be sleep deprived up to 6 months after hospitalisation (Liao, Huang, Huang, \& Hwang, 2011; Propper, van Domburg, Brunott, \& Bogers, 2015; Yang, Huang, Tsai, \& Lou, 2015).

In cardiac surgery, sleep disruption is characterised by the patient's inability to initiate and maintain sleep (Liao et al., 2011; Propper et al., 2015; Yang et al., 2015). Sleep disruption is a multidimensional phenomenon stimulated by a myriad of factors including, but not limited to, pain, anxiety, stress and inflammatory response postcardiopulmonary bypass (Casida, Davis, Shpakoff, \& Yarandi, 2014; Casida \& Nowak, 2011). Additionally, noise level and bright lighting practices are common environmental factors known to disrupt the sleep of patients recovering from cardiac surgery (Casida \& Nowak, 2011). Within the environments of care, however, the context of care provider interactions during patients' sleeping hours and the impact of such interactions on sleep and sleep outcomes in cardiac surgery are still understudied (Casida \& Nowak, 2011).

While night-time routine care interactions (NCRIs) have been proposed as a major environmental factor affecting the sleep of patients confined in acute and critical-care units (Bihari et al., 2012; Tamburri, DiBrienza, Zozula, \& Redeker, 2004), there exists a paucity of this information in the adult cardiac surgery unit. Through an extensive literature search, we found three studies that specifically addressed the influence of care interactions on sleep disturbances in cardiac surgery. Investigators in these studies, which were published from 1996 to 2012, had employed either retrospective (Simpson, Lee, \& Cameron, 1996) or cross-sectional research designs (Le et al., 2012; Makic, Rauen, Watson, \& Poteet, 2014) focusing on care interactions in critical-care units. These studies found significant relationships among procedures on patients, sleep effectiveness and sleep length. Although two studies (Simpson et al., 1996; Le et al., 2012) did not provide sleep data, investigators found care interactions were more frequent at midnight and less frequent at 3:00 a.m. Additionally, the frequency of care interactions in cardiac surgery critical care was significantly higher than the frequency of care interactions occurring in the general surgical, medical, paediatric and neonatal critical-care units (Let et al., 2012).

The objective of this study was to address the dearth of information about the context and the influence of the care environment on the patients' sleep in cardiac surgery units of hospitals. The specific

\section{What does this paper contribute to the wider global clinical community?}

- This study is the first empirical description of the type and frequency of NCRIs occurring during the first week following cardiac surgery.

- The study findings offer investigators a basis for examining the mechanism of the potential adverse effects of NCRIs on sleep and sleep outcomes following cardiac surgery. The knowledge that will be generated is crucial for designing an intervention research aimed at optimising patient's health and healthcare outcomes, postoperatively.

aims of this study were the following: (i) describe the type and frequency of NCRI during patients' sleeping hours (12 MN to 6:00 a.m.); (ii) determine whether the frequency of NCRI changes over the first postoperative week; (iii) describe the patients' perceptions of night-time sleep effectiveness (NSE) and daytime sleepiness/sleep supplementation (DSS) during the first postoperative week; (iv) examine the changes in the patients' perceptions of NSE and DSS over the first postoperative week; and (v) identify the relationships among NCRIs, NSE and DSS variables.

\section{2 | METHODS}

\section{1 | Design, sample and setting}

To address the objective of the study, we employed an exploratory repeated-measures design. Study patients were recruited and screened in an outpatient clinic using the following inclusion criteria: (i) first-time elective cardiac surgery using a cardiopulmonary bypass machine; (ii) male or female 18 years of age or older; (iii) able to read, write and understand written and verbal instructions in English; and (iv) provide informed consent. Patients were excluded from the study if they had a preoperative history or a diagnosis of a sleep disorder (e.g., sleep apnoea), substance abuse, neurologic or psychiatric disorders and used sleep medications, narcotics, anxiolytics and/or antidepressants. Of 215 patients screened, 38 provided an informed consent and completed the study. Recruitment and implementation of the study were conducted in the outpatient clinic and inpatient care units (critical- and progressive-care) of a large tertiary (urban) hospital in the Midwestern region of the USA. The study was approved by the affiliated university and hospital institutional review boards.

\section{2 | Data collection}

The patient demographics and clinical profiles were collected through interviews and reviews of medical records. The NCRI, NSE and DSS were collected in the critical- and progressive-care units. 
NCRIs were collected every night from the postoperative night (PON) 1 through PON 5. Patients' self-reported NSE and DSS collected upon awakening during postoperative days (POD) 1 through POD 5.

NCRIs were operationally defined as any type of patient carerelated activity (e.g., assessment, medication administration) implemented by the bedside RN or another healthcare provider (e.g., respiratory therapist, physician) during sleeping hours, from 12:00 MN to 6:00 a.m. During this time, no visitors are allowed, and the scheduled diagnostic tests (e.g., 12 lead ECG, chest radiograph) are not typically performed. Within this time frame, bedside RNs completed a Care Report Form on which the type of night-time care and frequency of care interactions were recorded.

The Verran Snyder-Halpern (VSH, 1990) Visual Analogue Sleep Scale (9 of 16 items) was used to measure night-time sleep and daytime sleepiness variables. Each item on this self-administered instrument consists of a 100-mm horizontal straight line where the ends of each line define the extreme limits of response being measured (e.g., "awoke exhausted" at the left end and "awoke refreshed" at the right end). The VSH scale has shown adequate reliability ( $\alpha=$ .70 to .93 ) and is sufficiently valid in measuring adult patients' perceptions of sleep and outcomes of sleep in hospitals and community settings (Casida et al., 2013; Snyder-Halpern \& Verran, 1987). The reliability coefficient derived from the sample of this study was .80 .

Sleep effectiveness is defined as the patient's perception of the effectiveness of his/her night-time sleep from the previous 24-hour period. Sleep effectiveness was measured by five scale items, including total sleep length (TSL) and sleep quality (SQ) variables. TSL is defined as the patient's perception of the total time spent in bed attempting to sleep and actually sleeping (i.e., bulk of sleep period). SQ is defined as the patient's perception about the adequacy of his/ her rest upon awakening and amount of sleep. Total mean TSL and SQ scores provide night-time sleep effectiveness (NSE) mean score. A higher NSE score implies sufficient sleep, while a lower score implies sleep disruptions (i.e., insufficient or poor night-time sleep). In normal or "healthy" sleepers, the mean and standard deviation $(S D, \pm)$ scores were as follows: $\mathrm{TSL}(M=80.4, S D=19.2)$, SQ $(M=66.5, S D=28.4)$ and NSE $(M=73.4, S D=23.8$ (Snyder-Halpern \& Verran, 1987; Verran \& Snyder-Halpern, 1990).

Daytime sleepiness is defined as the patient's perception of his/her daytime functioning manifested by the need for taking frequent naps during waking hours of the day to supplement the previous night's sleep loss. Daytime sleepiness was measured by four scale items from VSH, which assessed the temporal pattern of the patient's sleepiness throughout the day. The variables comprising this measure were as follows: (i) wake after final arousal (WAFA) - the initial morning awakening; (ii) morning sleep (AMS) - the amount of supplemental sleep during the morning hours; (iii) afternoon sleep (PMS) - the amount of sleep during afternoon hours; and (iv) daytime sleep (DTS) - the duration or time asleep outside of the primary (night-time) sleep period. The combined WAFA, AMS, PMS and DTS scores provide an overall daytime sleepiness or sleep supplementation score (DSS). A higher DSS score implies excessive daytime sleepiness, while a lower score implies alertness sustained during the day. In normal or "healthy" sleepers, the mean and SD scores were as follows: WAFA $(M=20.5, S D=28.7)$, AMS $(M=8.8$, $S D=20.5)$, PMS $(M=10.9, S D=22.9)$, DTS $(M=5.9, S D=13.6)$ and DSS $(M=11.5, S D=21.4)$ (Snyder-Halpern \& Verran, 1987; Verran \& Snyder-Halpern, 1990).

\section{3 | Data analyses}

We used SPSS IBM version 22.0 (IBM Corporation, 2013) for data management and analyses. NRCls were analysed by coding the patient care-related activity recorded by the RN on the Care Report Form, followed by applying descriptive analytics. Care activities were clustered into categories and coded as one type of NCRI (e.g., assessment). We used summary statistics (mean, SD or frequency distributions) to characterise the sample distribution of the study patients and to summarise the trend of NCRIs, NSE and DSS during the study period. Upon determining the normality of data ( -1.0 to 1.0 SD units) using the skewness coefficient formula (Munro, 2001), we inferred the changes over time in the mean scores of care interactions, night-time sleep and daytime sleepiness variables with repeated-measures analysis of variance (ANOVA) method. Furthermore, we used Pearson's correlations to estimate and test the relationships among the continuous variables of NCRIs, night-time sleep and daytime sleepiness. Type I error was set at 0.05 for all tests, and derived $p$-values were presented as the variable for the strength of evidence without multiple testing adjustment.

\section{RESULTS}

\section{1 | Patient characteristics}

The patient demographics and pertinent clinical characteristics are summarised in Table 1. Patients' mean age was 60 with SD of 15.9 years. Patients were predominantly male (60\%), married (66\%) and Caucasian (63\%). Most were educated beyond high school (45\%) and retired (37\%). The majority of the patients had a history of coronary artery disease (68\%), hypertension (68\%) and/or coronary artery bypass graft surgery (53\%). All patients had undergone standard operative procedures with the following intra-operative data: anaesthesia time $(M=359, S D=105 \mathrm{~min})$, cross-clamp time $(M=109.5$, $S D=69 \mathrm{~min})$, and bypass time $(M=139.2, S D=67 \mathrm{~min})$. Postoperative management included the administration of narcotic analgesics (97\%), beta-blockers (89\%) and vasoactive agents (71\%). The mean hospital length of stay was $7.1, S D=6.3$ days.

\subsection{Type and frequency of $\mathrm{NRCl}$}

The most frequent type of NCRIs occurring between midnight and 6:00 a.m. were a patient assessment (92\%), medication administration (87\%), laboratory/diagnostic procedures (78\%) and postoperative exercises (55\%). The least-frequent NCRIs during this time frame were multidisciplinary care rounds (14\%) and performing a procedure, such as intravenous catheter insertion (8\%) (Table 2). The 
TABLE 1 Patient demographics and clinical characteristics $(N=38)$

\begin{tabular}{|c|c|}
\hline Features & $\begin{array}{l}\text { Mean and SD } \\
n(\%)^{\mathrm{a}}\end{array}$ \\
\hline Age (mean in years) & $60 \pm 15.9$ \\
\hline \multicolumn{2}{|l|}{ Gender: } \\
\hline Male & $23(60.0)$ \\
\hline Female & $15(40.0)$ \\
\hline \multicolumn{2}{|l|}{ Marital status: } \\
\hline Single & $5(13.2)$ \\
\hline Married & $25(66.0)$ \\
\hline Domestic partner & $1(3.0)$ \\
\hline Divorced & $1(3.0)$ \\
\hline Widow & $5(13.2)$ \\
\hline \multicolumn{2}{|l|}{ Race/Ethnic background: } \\
\hline African American & $12(32.0)$ \\
\hline Asian & $2(5.3)$ \\
\hline Caucasian & $24(63.2)$ \\
\hline \multicolumn{2}{|l|}{ Education: } \\
\hline Some education & $3(8.0)$ \\
\hline High school & $5(13.2)$ \\
\hline Some college & 7 (18.4) \\
\hline College & 7 (18.4) \\
\hline Beyond college & $3(8.0)$ \\
\hline \multicolumn{2}{|l|}{ Employment status: } \\
\hline Full-time & $11(30.0)$ \\
\hline Part-time & $2(5.3)$ \\
\hline Retired & $14(37.0)$ \\
\hline Unemployed & $2(5.3)$ \\
\hline On disability & $2(5.3)$ \\
\hline \multicolumn{2}{|l|}{ Medical history: } \\
\hline Coronary artery disease/myocardial infarction & $26(68.4)$ \\
\hline Hypertension & $26(68.4)$ \\
\hline Hypercholesterolaemia & $19(50.0)$ \\
\hline Diabetes & $14(37.0)$ \\
\hline Valvular disease & 7 (18.4) \\
\hline Heart failure & $6(15.8)$ \\
\hline Pulmonary disease & $6(15.8)$ \\
\hline Other & $13(34.2)$ \\
\hline \multicolumn{2}{|l|}{ Surgical history: } \\
\hline Yes & $20(53.0)$ \\
\hline No & $18(47.0)$ \\
\hline \multicolumn{2}{|l|}{ Surgical procedure: } \\
\hline Coronary artery bypass graft (CABG) & $20(53)$ \\
\hline Valve replacement/repair & $16(42.1)$ \\
\hline CABG + valve repair & $2(5.3)$ \\
\hline Anaesthesia time (mean in min) & $359.0 \pm 105.0$ \\
\hline Cross-clamp time (mean in min) & $109.5 \pm 69.0$ \\
\hline
\end{tabular}

TABLE 1 (Continued)

\begin{tabular}{|l|l|}
\hline Features & $\begin{array}{l}\text { Mean and SD } \\
\mathrm{n}(\%)^{\mathrm{a}}\end{array}$ \\
\hline Bypass time (mean in min) & $139.2 \pm 67.0$ \\
\hline Postoperative medications: & \\
\hline Anxiolytics & $4(10.5)$ \\
\hline Beta-blockers & $34(89.5)$ \\
\hline Inotropic agents & $5(13.2)$ \\
\hline Narcotic analgesics & $37(97.4)$ \\
\hline Non-narcotic analgesics & $20(53.0)$ \\
\hline Sleep medications & $1(3.0)$ \\
\hline Vasoactive agents & $27(71.0)$ \\
\hline
\end{tabular}

"Because of rounding, missing or "not applicable" data or multiple responses, not all percentages total 100; SD (standard deviation).

frequency of NCRI ranged from 1 to 16 interactions every night, with the highest NCRI occurring on PON $1(M=4.5, S D=3.6)$. On PON 2 to 4 , the NCRIs were $M=2.8, S D=1.8 ; M=2.3, S D=1.6$; and $M=2.3, S D=1.4$, respectively (Table 3 ). The lowest NCRI was noted on PON $5(M=1.9, S D=0.9)$. Although there was a $62 \%$ decrease in NCRI between PON 1 and 2, and a gradual decrease between PON 3 to 5 , these reductions and changes in care frequencies over time were not statistically significant by repeated-measures ANOVA $(p=.72)$.

\subsection{Sleep effectiveness}

Table 4 is a summary of the patients' perceptions of their sleep effectiveness during the postoperative period. TSL scores ranged from $M=49.4, S D=12.1$ to $M=54.1, S D=14$ with lowest and highest scores on POD 3 and 5 , respectively. The respective lowest and highest scores of SQ were on POD 4 and POD 2. SQ scores ranged from $M 52.9, S D=31.8$ to $M=65.6, S D=21.3$. The trend in the patients' perceptions of their NSE was different from TSL and SQ. At POD 1, 2 and 5, NSE scores were somewhat the same, ranging from $M=57.7, S D=12.1$ to $M=57.8, S D=13.5$. The similarity

TABLE 2 Type of NCRI occurred between 12:00 MN and 6:00 a.m.

\begin{tabular}{|ll|}
\hline Night-time routine & $\mathbf{n}(\%)$ \\
\hline Assessment (e.g., vital signs, hemodynamics) & $35(92.1)$ \\
\hline Medication administration & $33(87.0)$ \\
\hline $\begin{array}{l}\text { Laboratory/diagnostic procedure (e.g., blood test) } \\
\text { Postoperative exercises (e.g., use of an incentive }\end{array}$ & $28(74.0)$ \\
\hline $\begin{array}{l}\text { spirometer) } \\
\text { Multidisciplinary care rounds }\end{array}$ & $21(55.3)$ \\
\hline Patient condition (e.g., arrhythmia) & $5(14.3)$ \\
\hline Equipment related (e.g., troubleshooting infusion pumps) & $5(14.3)$ \\
\hline Nursing procedure (e.g., intravenous catheter insertion) & $4(10.5)$ \\
\hline
\end{tabular}


of NSE scores, although lower, was also noted on POD 4 and 3 $(M=52.9, S D=17.2$ and $M=53.0, S D=14.4)$ than POD 2,3 and 6. Regardless of the variability in mean TSL, SQ and NSE scores, the changes in scores over time were not statistically significant by repeated-measures ANOVA; respective $p$ values were $.36, .81$ and .79 (Table 4).

\subsection{Daytime sleepiness}

A summary of the temporal pattern of daytime sleepiness among study patients is shown in Table 5 . The lowest WAFA score was at POD $1(M=20.8, S D=25.5)$, and the highest was at POD 3 $(M=44.4, S D=34.9)$, but these changes over time were not statistically significant by repeated-measures ANOVA $(p=.365)$. A distinct pattern and statistically significant changes in AMS, PMS and DTS scores were found throughout the postoperative period; respective $p$ values were $.004, .005$ and .003 . AMS scores ranged from $M=34.9$, $S D=32.4$ to $M=46.7, S D=36.4$ with the lowest score on POD 1 and highest score on POD 3. Most notably, the pattern of PMS, DTS and DSS scores was identical during POD 1 and 2. Respective lowest PMS and DTS scores were found during POD $1(M=28.3$, $S D=30.1$ and $M=24, S D=26.0$ ), and the highest scores were during POD $2(M=64.4, S D=32.9$ and $M=41.3, S D=41.3)$. The DSS scores ranged from $M=27, S D=22.6$ (POD 1) to $M=45.6$, $S D=16.5$ (POD 2), $p$ value $<.0001$ (Table 5).

\subsection{Relationship between NCRI, sleep effectiveness and daytime sleepiness}

Results of the pairwise association tests showed that only one NCRI (postoperative exercise) was significantly related to sleep variables: TSL $(r=.45, p=.042)$ and NSE $(r=.46, p=.035)$. No significant

TABLE 3 Frequency of NCRI between 12:00 MN and 6:00 a.m.

\begin{tabular}{|llll} 
Postoperative nights & Range & Mean & Standard deviation $( \pm)$ \\
\hline 1 & $1-16$ & 4.5 & 3.6 \\
\hline 2 & $2-11$ & 2.8 & 1.8 \\
\hline 3 & $1-11$ & 2.3 & 1.6 \\
\hline 4 & $1-10$ & 2.3 & 1.4 \\
\hline 5 & $1-6$ & 1.9 & 0.9 \\
\hline
\end{tabular}

TABLE 4 Repeated-measures ANOVA of patients perceptions of night-time sleep $(N=38)$ relationship between NCRI and daytime sleepiness variables were found. Using the same approach, we also found that there was no significant relationship between TSL and daytime sleep variables. However, we found that SQ was negatively related to DTS $(r=-.42$, $p=.008)$ and DSS $(r=-.41, p=.010)$. Also, NSE was negatively related to DTS $(r=-.49, p=.002)$ and DSS $(r=-.44, p=.006)$.

\section{4 | DISCUSSION}

We found eight types of NCRIs commonly occurring during the sleeping hours of patients in cardiac surgery units of a hospital. The findings suggested that patients were awakened by the RN or other healthcare provider at least once every hour on PON 1 and every 2 to 2.5 hrs on PON 2 to 5, between the hours of $12 \mathrm{MN}$ and 6:00 a.m. This assertion was based on the data on the Care Report Form documenting the reasons of "purposely waking patients" during these hours. Major reasons for waking patients were to perform assessments, medication administration and laboratory tests, which were consistent with previous research (Let et al., 2012). However, the reduction in the frequency of NCRIs over time (albeit not statistically significant) is a new finding that merits close attention and future investigation. This nonsignificant finding may be explained by clinical stability, as the majority of the patients were transferred out of the critical-care into the progressivecare units between POD 3 and 5 .

Despite the reduction in NCRIs, patients' sleep was disrupted throughout the first postoperative week. Their sleep disruptions are shown by the nonstatistically significant change in their TSL, SQ and NSE mean scores (Table 3). These scores are lower than the scores of "healthy sleepers" in previous VSH studies (Verran \& Snyder-Halpern, 1990), which suggest ineffective or insufficient sleep (i.e., disrupted sleep) when compared to norms. This assertion, however, requires further investigation as the mean age $(M=60.0$, $S D=15.9$ years) of our sample is older than the mean age ( $M=39.5, S D=10.4$ years) of the comparative healthy sleepers. Sleep is expected to change with ageing. As the person ages, sleep becomes more shallow with much less deep sleep (Verster, PandiPerumal, \& Streiner, 2008). Because of the nonsignificant changes in NCRI frequencies, TSL, SQ and NSE mean scores (Tables 2a to 4), we speculated that NCRIs might have also contributed to a myriad of factors (e.g., pain and anxiety) that are known to disrupt sleep in cardiac surgery (Casida \& Nowak, 2011). However, the degree to which

\begin{tabular}{lllllll}
\multicolumn{7}{c}{ Mean scores and standard deviations $( \pm)$} \\
\cline { 2 - 8 } Variables & POD 1 & POD 2 & POD 3 & POD 4 & POD 5 & $\begin{array}{l}\text { v- } \\
\text { value }\end{array}$ \\
Total sleep length (TSL) & $50.8(17.8)$ & $51.7(11.4)$ & $49.4(12.1)$ & $52.9(31.8)$ & $54.1(14.0)$ & .36 \\
Sleep quality (SQ) & $64.0(24.2)$ & $65.6(21.3)$ & $56.0(25.6)$ & $52.9(31.8)$ & $61.4(22.8)$ & .81 \\
\hline $\begin{array}{l}\text { Night-time sleep } \\
\text { effectiveness (NSE) }\end{array}$ & $57.7(17.5$ & $57.7(12.1)$ & $53.0(14.4)$ & $52.9(17.2)$ & $57.8(13.5)$ & .79 \\
\hline
\end{tabular}

Normal values $(\mathrm{TSL}=80.4 \pm 19.2, \mathrm{SQ}=66.5 \pm 28.4$ and NSE $=73.4 \pm 23.8$ ) 


\begin{tabular}{|c|c|c|c|c|c|c|}
\hline \multirow[b]{2}{*}{ Variables } & \multicolumn{6}{|c|}{ Mean scores and standard deviations $( \pm)$} \\
\hline & POD 1 & POD 2 & POD 3 & POD 4 & POD 5 & $\begin{array}{l}p- \\
\text { value }\end{array}$ \\
\hline $\begin{array}{l}\text { Wake after final arousal } \\
\text { (WAFA) }\end{array}$ & $20.8(25.5)$ & $31.6(29.0)$ & $44.4(34.9)$ & 39.9 (36.1) & $35.8(33.2)$ & .365 \\
\hline Morning sleep (AMS) & $34.9(32.4)$ & $45.1(33.5)$ & $46.7(36.4)$ & $39.2(35.4)$ & $42.7(33.8)$ & .004 \\
\hline Afternoon sleep (PMS) & $28.3(30.1)$ & $64.4(32.9)$ & $49.5(34.9)$ & $47.7(36.6)$ & $48.0(33.6)$ & .005 \\
\hline Daytime sleep (DTS) & $24.0(26.0)$ & $41.3(23.6)$ & $39.1(24.6)$ & $24.1(22.9)$ & $31.2(24.5)$ & .003 \\
\hline $\begin{array}{l}\text { Daytime sleepiness/ } \\
\text { sleep supplementation } \\
\text { (DSS) }\end{array}$ & $27.0(22.6)$ & $45.6(16.5)$ & $44.8(22.1)$ & $37.7(21.4)$ & $39.7(24.9)$ & $<.0001$ \\
\hline
\end{tabular}

TABLE 5 Repeated-measures ANOVA of patients perceptions of daytime sleepiness $(N=38)$

Normal values $(\mathrm{WAFA}=20.5 \pm 28.7, \mathrm{AMS}=8.8 \pm 20.5, \mathrm{PMS}=10.9 \pm 22.9$, DTS $=5.9 \pm 13.6$ and

$\mathrm{DSS}=11.5 \pm 21.4)$.

NCRIs and these factors influence sleep, specifically the intensity and the frequency of postoperative exercises on sleep and sleep outcomes (e.g., daytime sleepiness), warrants further research.

The significant associations among postoperative exercise, TSL and NSE infer that frequent postoperative exercises during the defined sleeping hours contribute to the decrease in TSL and NSE scores. This assertion is supported by the fact that patients' in the present study had lower TSL and NSE scores than normative sample (Verran \& Snyder-Halpern, 1990). Nonetheless, NCRIs are amenable to intervention. Le et al. (2012) reported that critical-care RNs were comfortable omitting some NCRIs without compromising patient outcomes. According to the RNs, $16 \%$ of routine assessment, $14 \%$ of routine care activities and $10 \%$ of night-time interventions can be safely omitted between 10:00 PM and 6:00 a.m. (Let et al., 2012). Thus, routine postoperative exercises (e.g., use of incentive spirometry) could be deferred from 10:00 p.m. until 6:00 a.m. to promote patient's night-time sleep in cardiac surgery units.

While the practice of clustering NCRIs (e.g., bed bath) has been implemented in some critical-care units as a sleep promotion intervention (Bihari et al., 2012), the extent of this practice in cardiac surgery units remains unknown. To fill this knowledge gap, research should include mapping and match the necessity and frequency of NCRIs with the patient's clinical stability and physiological integrity. This research can be accomplished by clinical assessments or perhaps risk stratifications, similar to what has been proposed for nonsurgical patients. For example, in a study involving 54,096 hospitalised patients, Yoder, Yuen, Churpek, Arora, and Edelson (2013) suggested that night-time vital sign monitoring may be safely omitted in medically stable patients (low risk) based on the Modified Early Warning Score (MEWS). With the goal of promoting sleep and facilitating physiological repair during recovery and rehabilitative periods, investigators should attempt to examine the feasibility and usability of MEWS (a risk stratification model) ${ }^{22}$ in adult cardiac surgery by starting with select "stable patients" in progressive-care units. This can be a starting point to challenge the traditional 24-hour regimen of routine vital signs monitoring and administration of medications.

Although only one NCRI was associated with sleep variables, the extent of the patients' daytime sleepiness throughout the waking hours of the day was uncovered by the present study. Mean scores presented in Table 4 are far higher than the normal/healthy sleepers (Verran \& Snyder-Halpern, 1990), suggesting excessive sleepiness throughout the day. The data also inferred that during POD 2, daytime sleepiness was far more excessive than PODs 1, 3, 4 and 5. Excessive daytime sleepiness is a common consequence of insufficient sleep among adults with various illnesses and conditions. It can impair physical and mental function, as well as the quality of life (Buysee, 2014; Verster et al., 2008). Notably, the significant associations among SQ, NSE, DTS and DSS suggest that patients required frequent naps beyond sleeping hours to compensate for sleep loss. Naps occurred in the early morning until late afternoon hours. Although daytime sleepiness is understudied in cardiac surgery, excessive naps during the day can further impair daytime functioning. Potential hazards of excessive daytime sleepiness may include, but are not limited to, increased risks for postoperative complications prevented by frequent ambulation (e.g., deep vein thrombosis, pressure ulcers) and pulmonary exercises (e.g., atelectasis, pneumonia) (Casida \& Nowak, 2011).

\section{1 | Limitations and future directions}

The main limitations of this study include the omission of sleep fragmentation items of the VSH scale, which may have further explained the patients' perceptions of sleep disruptions. The use of self-report measures and nonrandom sampling procedures were additional limitations. Thus, the interpretation of the results should be confined within the limits of the study aims and use of selfreport measures that can be influenced by several factors leading to underestimation or overestimation of the results (Casida et al., 2014). We acknowledged that patients who had taken anxiolytics, beta-blockers and/or sleep medications (Table 1) might have biased perceptions of their sleep quality as these medications are known to disrupt or promote sleep (Vallerand \& Sanoski, 2014). Also, our sample from a single centre may not be an accurate representation of adult cardiac surgery population in the USA and the world, thus limit the generalisability of the results. However, despite the 
limitations, we hope our data encourage researchers and clinicians to continually investigate the traditional care delivered at night in adult cardiac surgery.

Future research is needed to address the study limitations to form a definitive conclusion, particularly the effect of NCRIs on sleep disruptions in adult cardiac surgery. Demographics (e.g., age) and other variables that are known to disrupt (e.g., pain and anxiety, beta-blockers) or promote (e.g., sleep medications) should be collected and considered as confounding variables in future research designs. Research should be implemented in multiple centres including a diverse, random sample of cardiac surgery procedures (e.g., reoperations, ventricular assist devices, and heart/lung transplants) in community, tertiary and quaternary heart centres. However, there have been studies in other patient care units regarding NCRIs and sleep research in cardiac surgery is still warranted due to the differences in clinical trajectory (i.e., exposure to cardiopulmonary bypass) and outcomes that are possibly sensitive to excessive daytime sleepiness, resulting in delayed ambulation-related complications described previously. Also needed is a rigorous research design using an objective measure of sleep (e.g., polysomnogram) (Verster et al., 2008) and a camera to capture NCRIs and patients' clinical or physiological needs in "real-time." These future studies would expand our knowledge of the context and the need for challenging the traditions of care routinely implemented during sleeping hours in cardiac surgery units. By objectively matching NCRIs with physiological and clinical states, as well as assessing the risks and benefits associated with the omission of some NCRIs during sleeping hours, we could produce mechanistic data needed for developing and testing sleep promotion interventions.

\section{5 | CONCLUSION AND RELEVANCE TO CLINICAL PRACTICE}

NCRIs appeared to decrease over the first postoperative week. However, patients still experienced sleep disruptions and excessive daytime sleepiness. Research is needed to explicate the cause and effect of NCRI on sleep disruption, daytime function and clinical outcomes. This would guide the development and testing of interventions aimed at improving both health and healthcare outcomes in diverse patient populations in various types of hospitals. Acute and critical-care nurses in the world are well positioned to lead the advancement of sleep promotion science and to transform the nighttime care delivery processes in adult cardiac surgery.

\section{CONTRIBUTIONS}

Study design: JMC, JED; Data collection and analysis: JMC, AZ, JJY; Manuscript preparation: JMC, JED, AZ, JJY.

\section{ORCID}

Jesus M Casida iD http://orcid.org/0000-0001-6342-8504

\section{REFERENCES}

Bihari, S., McEvoy, R., Matheson, E., Kim, S., Woodman, R. J., \& Bersten, A. D. (2012). Factors affecting sleep quality of patients in intensive care unit. Journal of Clinical Sleep Medicine, 3(15), 301-307.

Bojar, R. M. (2011). Manual of perioperative care in adult cardiac surgery (5th ed.). West Sussex, UK: Wiley-Blackwell.

Buysee, D. J. (2014). Sleep health: Can we define it? Does it matter? Sleep, 1(37), 9-17. https://doi.org/10.5665/sleep.3298

Casida, J. M., Davis, J. E., Shpakoff, L., \& Yarandi, H. (2014). An exploratory study of the patients' sleep patterns and inflammatory response following cardiopulmonary bypass (CPB). Journal of Clinical Nursing, 23, 2332-2342. https://doi.org/10.1111/jocn.12515

Casida, J. M., \& Nowak, L. (2011). Integrative therapies to promote sleep in the ICU. In L. Chlan \& M. Herts (Eds.). Integrated therapies in lung health \& sleep. (pp. 177-187). New York, NY: Springer.

Casida, J. M., Yaremchuk, K. L., Shpakoff, L., Marrocco, A., Babicz, G., \& Yarandi, H. (2013). The effects of guided imagery on sleep and inflammatory response in cardiac surgery: A pilot randomized controlled trial. Journal of Cardiovascular Surgery, 2(54), 269279.

Friese, R. S. (2008). Sleep and recovery from critical illness and injury: A review of theory, current practice, and future directions. Critical Care Medicine, 3(36), 697-705. https://doi.org/10.1097/CCM.0B013E3 181643F29

IBM Corporation (2013). IBM SPSS Statistics for Windows Version 22. Armok, NY: IBM Corporation.

Le, A., Friese, R. S., Hsu, C. H., Wynne, J. L., Rhee, P., \& O'Keefe, T. (2012). Sleep disruptions and nocturnal nursing interactions in the intensive care unit. Journal of Surgical Research, 2(177), 310-314. https://doi.org/10.1016/j.jss.2012.05.038

Liao, W. C., Huang, C. Y., Huang, T. Y., \& Hwang, S. L. (2011). A systematic review of sleep patterns and factors that disrupt sleep after heart surgery. Journal of Nursing Research, 4(4), 275-288. https://doi.org/ 10.1097/JNR.0b013e318236cf68

Makic, M. B., Rauen, C., Watson, R., \& Poteet, A. W. (2014). Examining the evidence to guide practice: Challenging practice habits. Critical Care Nurse, 2(34), 28-45. https://doi.org/10.4037/ccn2014262

Munro, B. H. (2001). Statistical methods for health care research (4th ed.). Philadelphia, PA: Lippincott.

Propper, J., van Domburg, R. T., Brunott, M., \& Bogers, A. J. J. C. (2015). Quality of sleep at the ward after cardiothoracic surgery. Open Journal of Nursing, 5, 529-537. Retrieved from http://file.scirp.org/pdf/ OJN_2015061216341548.pdf. https://doi.org/10.4236/ojn.2015. 56056

Simpson, T., Lee, E. R., \& Cameron, C. (1996). Relationships among sleep dimensions and factors that impair sleep after cardiac surgery. Research in Nursing and Health, 3(9), 213-223. https://doi.org/10. 1002/(ISSN)1098-240X

Snyder-Halpern, R., \& Verran, J. A. (1987). Instrumentation to describe subjective sleep characteristics in healthy subjects. Research in Nursing and Health, 3(10), 155-163. https://doi.org/10.1002/(ISSN)1098$240 \mathrm{X}$

Tamburri, L. M., DiBrienza, R., Zozula, R., \& Redeker, N. S. (2004). Nocturnal care interactions with patients in critical care units. American Journal of Critical Care, 2(13), 102-112.

Thompson, D. R., Hamilton, D. K., Cadenhead, C. D., Swoboda, S. M., Schwindel, S. M., Anderson, D. C., Schmitz, E. V., ... Petersen, C. (2012). Guidelines for intensive care unit design. Critical Care Medicine, 5(40), 1586-1600.

Vallerand, A. H., \& Sanoski, C. A. (2014). Davis' drug guide for nurses (14th ed.). Philadelphia, PA: F. A. Davis Company.

Verran, J. A., \& Snyder-Halpern, R. (1990). Visual analog sleep scales. Tucson, AZ: University of Arizona College of Nursing. 
Verster, J. C., Pandi-Perumal, S. R., \& Streiner, D. L. (2008). Sleep and quality of life in clinical medicine. Totowa, NJ: Humana Press. https://doi.org/10.1007/978-1-60327-343-5

Yang, P. L., Huang, G. S., Tsai, C. S., \& Lou, M. F. (2015). Sleep quality and emotional correlates in Taiwanese coronary artery bypass grant patients 1 week and 1 month after hospital discharge: A repeated descriptive correlational study. PLoS ONE, 10(8), e0136431. Retrieved from http://journals.plos.org/plosone/article?id=10.1371/journal. pone.0136431

Yoder, J. C., Yuen, T. C., Churpek, M. M., Arora, V. M., \& Edelson, D. P. (2013). A prospective study of night-time vital sign monitoring frequency and risk of clinical deterioration. Journal of American Medical Association Internal Medicine, 2173, 1554-1555.

How to cite this article: Casida JM, Davis JE, Zalewski A, Yang JJ. Night-time care routine interaction and sleep disruption in adult cardiac surgery. J Clin Nurs. 2018;27: e1377-e1384. https://doi.org/10.1111/jocn.14262 